\title{
Palliative Care in the ICU
}

\author{
Moderator: Judith E. Nelson, M.D., J.D., \\ Participants: Elie Azoulay, M.D., Ph.D., ${ }^{2}$ J. Randall Curtis, M.D., M.P.H., ${ }^{3}$ Anne C. Mosenthal, M.D., FACS, ${ }^{4}$ \\ Colleen M. Mulkerin, M.S.W., LCSW, ${ }^{5}$ Kathleen Puntillo, R.N., DNSc., FAAN, ${ }^{6}$ and Mark D. Siegel, M.D.?
}

Judith E. Nelson, M.D., J.D. (Moderator): I am pleased to have this opportunity to have a roundtable discussion about palliative care in the intensive care unit with a group of my distinguished colleagues. You are a diverse interdisciplinary group, from various geographical areas around the United States and across the ocean in Europe, and all experts on this topic. Also, all of you, with the exception of Dr. Siegel, are members of the advisory board of The IPAL-ICU Project, which is supported by the National Institutes of Health and the Center to Advance Palliative Care.

I would like to open up this discussion by asking members of the group to comment on why the ICU is such an important venue for providing, improving and evaluating palliative care. Perhaps we can begin with Dr. Curtis.

J. Randall Curtis, M.D., M.P.H.: Sure. There has been a lot of interest in improving palliative and end-of-life care in the ICU for the past several decades for a number of reasons. One is that in the ICU we care for the sickest patients. These patients are often at high risk of dying and require the ICU either for life support or intensive monitoring. Even if they survive, critically ill patients may have significant sequelae that can permanently affect their quality of life, and cause symptoms and other problems. Therefore, palliative care is an important component of ICU care for many patients.

Judith E. Nelson, M.D., J.D.: Thank you. I wonder if others at this roundtable would like to comment further on this issue about the central role of the ICU for the field of palliative care and its improvement.

Mark D. Siegel, M.D.: Even though the needs in palliative care have been apparent to many people for a long time, there has been a significant shift in focus in the critical care community with increasing appreciation of the needs of ICU patients and families. Looked at today, the palliative care needs in the ICU are actually classic and akin to the needs of patient populations that were traditionally recognized as requiring palliative care.

In addition to ICU patients being at high risk of death and having symptoms, these patients are also accompanied by family members who are often suffering and need psychological support and multidisciplinary support, not just from the physicians, but from nurses, pastoral caregivers, and social workers. So the multidisciplinary effort that works very well in other settings, such as the oncology setting, is essential in the ICU setting as well.

Elie Azoulay, M.D., Ph.D.: In Europe, the ICU is really the only place where we are providing palliative care. Most European hospitals do not have mobile teams of palliative care professionals. We also do not have many specialists providing palliative care outside or inside the ICU. So we can only rely on the ICU and ourselves. Again, the multidisciplinary approach is very valuable, and the ICU, with this approach, may be the best place that we can provide palliative care. Occasionally, intensivists are called to provide help with palliative care on the wards.

In 2005, France passed a law stating that palliative care was a priority. Since then, people are getting trained in palliative care. But this is only in oncology and hematology departments. For elderly patients, those with serious conditions and for many terminal conditions and situations, palliative care is beneficial and ICU clinicians are called to help. ICU residents and the fellows are trained in the ICU to become the "specialists of life", but they are also becoming specialists in endof-life care.

Judith E. Nelson, M.D., J.D.: That is a very interesting point about the different models for providing palliative care in the ICU, and we will come back to that. Because of the situation that you face in Europe, it becomes necessary to have internal palliative care capability within your ICU teams and that is still the case in some hospitals and ICUs in the United States.

\footnotetext{
${ }^{1}$ Division of Pulmonary, Critical Care and Sleep Medicine, The IPAL-ICU Project, Mount Sinai School of Medicine, New York, New York.

${ }^{2}$ Service de Réanimation Médicale Groupe de Recherche Respiratoire en Réanimation Onco-Hématologique Groupe de Recherche Famiréa Hôpital Saint-Louis, Université Paris VII Paris, France.

${ }^{3}$ Departments of Medicine and Pulmonary and Critical Care Medicine, Harborview Medical Center, University of Washington, Seattle, Washington.

${ }^{4}$ Department of Surgery and Division of Surgical Critical Care, UMDNJ-NJMS, Newark, New Jersey.

${ }^{5}$ Department of Palliative Medicine, Hartford Hospital, Hartford, Connecticut.

${ }^{6}$ Department of Physiological Nursing University of California, San Francisco, San Francisco, California.

${ }^{7}$ Pulmonary and Critical Care Section, Department of Internal Medicine, Yale School of Medicine, Yale-New Haven Hospital, New Haven, Connecticut.
} 
I would also like to point out that although we have made a lot of strides in terms of addressing the needs of patients and families in the ICU for palliative care, this is a problem that is increasing in scope. We have, for example, the findings of a recent report on end-of-life care from the Dartmouth Atlas Project, which has done a lot of work comparing trends in care for Medicare beneficiaries in different areas of the United States. This report shows that in 2007, as compared to four or five years earlier, Medicare patients who have severe chronic illnesses are spending fewer days in the hospital, are less likely to die in hospital, and are more likely to receive hospice care. Yet, those who are in the hospital are actually spending more days in intensive care units. So we have a growing population of elderly patients and a growing number of chronically ill patients receiving intensive care. Therefore, the ICU becomes only more important as a venue as we look to the future.

I would like to move on to discussion of what you see as special challenges for providing and improving palliative care in the ICU setting. I would like to begin that conversation with Colleen Mulkerin, who has broad experience providing palliative care in a variety of venues, including the ICU. Colleen?

Colleen M. Mulkerin, M.S.W., LCSW: One of the things that we face in the ICU environment is the need to balance the specialty interests of the primary critical care team and all the good work that is happening in the ICU, with the interests of specialists coming from palliative consult teams. Having those models work together can be very helpful. Some of the ICU clinicians are very proud and protective of the work they are doing in the ICU and the challenge of having a consultative service come in can be quite difficult. Trying to support and integrate some of the palliative care concepts and practices into what the critical care teams are doing can be so valuable. With a little bit of support and education, we as palliative care specialists are able to improve the ability of the ICU team to do family meetings, to involve families on rounds, and develop better ways to integrate patients' values and preferences for care.

It can also be quite challenging to make sure that you are sensitive to the culture of the specific ICU - who the patient population is, whether you have an open or closed unit really doing an assessment and customizing the work for that particular ICU (whether it be the surgical or medical ICU), considering what has been and will be successful in that place.

Judith E. Nelson, M.D., J.D.: Those are very important points, and I wonder if Dr. Mosenthal wants to comment on some of the special challenges that you have addressed in the context of the surgical intensive care unit.

Anne C. Mosenthal, M.D., FACS: The surgical population in the ICU has some particular challenges, although I think each specialty ICU has particular challenges. In the surgical ICU, there are some structural issues. Colleen, you mentioned the issue of open versus closed ICUs, and that tends to be a challenge in a lot of surgical ICUs. In open ICUs, there is not necessarily a multidisciplinary team approach to critical care, and that impedes the ability to improve palliative care as well.
In many surgical ICUs, depending on the specialty, the focus is on "rescue". There is less willingness to acknowledge some patients may die, and that palliative care is necessary. This is a challenge, both in terms of surgeons and other health care providers in a surgical ICU.

In certain ICU populations with a high mortality rate, such as liver transplant or trauma patients, there may still be a lack of appreciation that end-of-life care is an important part of the care in the ICU, needing special attention.

Judith E. Nelson, M.D., J.D.: Dr. Mosenthal, you have been very helpful in pointing out some of the categories of special issues that may arise in the surgical intensive care unit setting. I know that this is an area in which you and other members of the advisory board of The IPAL-ICU Project of the Center to Advance Palliative Care have been working, and have recently published an article providing some guidance addressing some of these special issues. Do others want to comment here? It would be very helpful to hear from Kathleen Puntillo about special challenges in the ICU for palliative care from a nursing perspective.

Kathleen Puntillo, R.N., DNSc., FAAN: I have two thoughts: The first is that we still oftentimes maintain this very narrow focus that sees palliative care only as end-of-life care, and if the patient is not deemed to be at the end of life, then it is not time to call in palliative care services. This narrow focus prevents patients and their families from getting the different types of palliative care that are available when we look at palliative care as something that all ICU patients should have access to.

My second thought about this is that if we focus on one very important aspect of palliative care, and that is symptom management, I think there are great challenges in ICUs and for ICU patients if we are not at the point where we are saying this patient is imminently dying. We have this tension, if you will, between the patient's physiological instability and the need for symptom management. Indeed, many patients in ICUs suffer from many symptoms such as fatigue, thirst, anxiety, and pain, so they have a need for symptom assessment and management. But again, this need for interventions or thoughts of interventions for symptoms often gets waylaid by the concern that we are going to make patients physiologically unstable.

Mark D. Siegel, M.D.: I want to first of all say that what Kathleen says really resonates with me in the ICU. I think that the needs of the ICU patient population, even if patients are not dying, are incredibly important.

There are a couple of practical issues. One has to do simply with staffing. At my institution, we do not have the largest palliative care service in the world, and at the moment, the resources are largely being directed towards the oncology population. For the non-oncology population, and in particular for the ICU population, there just is not enough time in the day for our palliative care physicians and nurse practitioners to see all the patients who have needs. There is a need to convince hospital administrators to make the investment in palliative care personnel, so that when the ICU physicians and nurses recognize the need, the palliative care experts will actually be there to provide those services. Fortunately, my institution, Yale-New Haven Hospital, is now making a 
major investment in resources and personnel to ensure that patients throughout our hospital get the palliative care services they need.

The other thing that Kathleen said that I would like to highlight is that we believe, but we do not really know for sure, that there is tension between symptom management and lifesaving interventions and management. I actually think that there is a need for a great deal more research to determine whether or not providing good symptom control really undermines in any way the larger goals of trying to save a patient's life. It may be true under some circumstances, and then under other circumstances I could easily imagine that if you can address patient's palliative care needs that they may actually do better.

If they sleep well, if their pain is under control, if they are less anxious, perhaps there will be other benefits to them clinically as well. It would be very helpful for us to have more research regarding this perceived tension and to see where palliative care actually fits into the overall goal of care in managing these patients.

Judith E. Nelson, M.D., J.D.: You bring up a really important point, which is the challenge that we face of a traditional view that persists in seeing palliative care and intensive care as mutually exclusive forms of care rather than mutually enhancing forms of care. We are beginning to see data coming out of not only the critical care arena, but other areas indicating that early integration of palliative care and management may actually prolong patients' lives.

One of the points that came up was that not all levels of hospital and ICU management understand the importance of investing in high-quality palliative care for all patients in the intensive care unit, whether it is provided through a palliative care consultation service, which may need additional resources to meet those needs, or whether it is provided through the internal capabilities of the ICU, or what is perhaps the most effective model, which is some combination of both.

I would point out at this point that The IPAL-ICU Project website at www.capc.org/IPAL-ICU includes a PowerPoint presentation entitled, "Making the case for ICU palliative care." The presentation outlines how integration of palliative care has been extremely successful in reducing ICU length of stay and hospital length of stay for ICU patients without increasing mortality, while at the same time providing good symptom control, high patient and family satisfaction, and improved psychological well-being of families both in the ICU and after. I wonder what others might want to contribute about making a case for the importance of investing in palliative care in the intensive care unit.

Mark D. Siegel, M.D.: This has been a hot topic at Yale, and I was very happy to hear the director of our cancer center saying that we should admit right at the outset that it is just the right thing to do, that patients expect it of us, that we should be providing good palliative care, and that regardless of cost and length of stay issues, if you want to provide excellent care, you must include a palliative care domain.

Now, the people who do the accounting and keep the finances might like to have a good financial model, and I think it is impossible to ignore that. But I want to be one to say up front that I think we also are in the business of just providing good care.
Elie Azoulay, M.D., Ph.D.: One of the problems in Europe is that in most ICUs, length of stay is not really a relevant outcome, because we cannot discharge a dying patient to the wards. Most other specialists would not agree to care for a dying patient, and since we do not have palliative care specialists, there is a real problem. Most of the patients who are dying in the ICU actually die in the ICU. They cannot be discharged to die in a peaceful setting rather than in the ICU.

Judith E. Nelson, M.D., J.D.: Yes, palliative care capability and this includes the ICU - is a standard of quality and excellence in the United States and, increasingly, overseas. This is a core service on the American Hospital Association survey. It is a criterion in the US News \& World Report's ratings of America's best hospitals. It is recommended as a requirement for cancer center certification, and the Joint Commission has introduced an advanced certification program recognizing palliative care excellence. So I think you are right, that palliative care, apart from all these other benefits, has become a standard of quality and excellence for ICUs and hospitals.

I would like to pursue the issue about the valuable roles that nurses in particular can play in an ICU palliative improvement effort. Kathleen, as a nursing representative on this panel, I wonder if you could address this.

Kathleen Puntillo, R.N., DNSc., FAAN: If I could expand that question just a little bit, I would like to reword it to, what are the responsibilities of nurses and their valuable roles? Because I think in many respects if nurses really are reminded of their responsibilities to be active members of all aspects of patient palliative care, then I think this gives them a framework from which to draw some confidence. By that I mean that if you look at any of the major professional nursing organizations, the American Nurses Association, the American Association of Critical Care Nurses and some of the European nurses' organizations, over and over again they emphasize that the nurse's primary commitment to the patient is not just a right but a responsibility, this one duty trumps all others. We really must be more involved in palliative care. I think that lays a good foundation for nurses.

Of course, the roles that nurses play are extremely important roles. Nurses are generally competent to take on a larger role, to discuss patient status with patients and families since they have more contact with patients than other clinicians in the ICU. They work frequently with dying patients. They hear discussions and conversations among family members, patients and multiple teams. So they do have the ability and the exposure to be involved.

Now, to be a little bit more specific, nurses can, for example, be the leaders in interdisciplinary committees or work groups to improve palliative care efforts. They can easily, and often do, identify potential obstacles for improving palliative care in their particular ICU. They can be leaders in enhancing teamwork that is so important to palliative care interventions and efforts. They can identify work processes that will help to integrate palliative care interventions and so on and so forth. So nurses have tremendous roles to play.

Within a family conference in which patients and families interests and needs are discussed, there are many specific things that nurses can do such as listening, facilitating, supporting the expression of empathy, and helping after the 
meeting to continue those conversations and clarify with other health care providers, as well as with family members who may not have heard everything during that meeting.

Finally, I want to make one more point that nurses can really guarantee a patient's dignity or their right to respect and ethical treatment, because a lot of times patient dignity is lost due to their dependency, their symptom distress, privacy boundaries, and their feeling of being a burden to others. Nurses have a tremendous responsibility and right and role in this whole aspect of palliative care.

Judith E. Nelson, M.D., J.D.: Would others at the roundtable like to speak on this issue about interdisciplinary roles, and specifically those of the nurse or other clinicians in addition to physicians in the ICU?

J. Randall Curtis, M.D., M.P.H.: I would just like to add to what Kathleen said to make the point that in ICUs where there is good interdisciplinary collaboration and communication between physicians and nurses, then nurses are in a position, as Kathleen pointed out, to be pivotal in reinforcing communication, ensuring that patients' and families' needs are met, and improve the overall communication between the team and the family. However, in ICUs where the communication and collaboration between the physicians and the nurses is not good, not only do you lose that opportunity, but actually it can make the patient and family experience much worse. Families may feel like they are hearing different messages from different members of the team and that they are getting mixed messages or conflicting stories. That is one of the most distressing things that family members, as well as patients, experience in the ICU.

Elie Azoulay, M.D., Ph.D.: One of the results of the last surveys of ICU nurses in France is that nurses are not as concerned about the decision-making process itself, but they are concerned that what is being done for the patient is not the same as what has been decided. The nurses are much more present, and they are taking part in the decision, but they would like to see the decisions really applied to patient care, to be sure that the way we are implementing the care for dying patients is what has been decided.

Anne C. Mosenthal, M.D., FACS: I wanted to talk about the model at our hospital, which is a communication model incorporating palliative care counselors into routine ICU care. Counselors facilitate the family meetings and communications, particularly around the end of life. At least in our setting, they have taken on the role that you have described, Kathleen, in terms of empowering families to ask questions and helping them in areas where they may not have remembered or heard all the information. The counselors also provide an educational role for the nurses and the physicians, which I think is really invaluable. So it is just a slightly different model for other disciplines to be involved in palliative care in the ICU.

Judith E. Nelson, M.D., J.D.: Anne, you have been able to make this work extremely successfully in a surgical intensive care unit setting, which was quite a pioneering effort and has demonstrated what is possible when there is strong interdisciplinary collaboration between nurses, physicians, chaplains and others toward this goal in any setting, and that is really very important.

Colleen, I wonder if you, as both a social worker and the director of a palliative care consultation service, might talk about the integration of disciplines to improve palliative care, both the disciplines within the ICU team and the disciplines of palliative care and critical care. You are running one of the busiest palliative care consultation services in America, if not the world, and perhaps you could provide some guidance on how that works best in an interdisciplinary way.

Colleen M. Mulkerin, M.S.W., LCSW: As I was listening to Kathleen, I was thinking that when you use all the resources on the team, those times when your patient is unstable and nursing is not able to be available to the family because of the primary duty to care for the patient, other members of the team like the social worker or the palliative consult service can step in to be a part of some of those longer counseling opportunities or conversations. Also, when the family system is quite complex, having an additional support like a clinical social worker to tease out the legal surrogates and to do the complex family systems work that sometimes is needed in critical care environments, those contributions can be really helpful.

The other thing that we have done with our palliative medicine team is to focus on the ICU palliative care quality indicators in the Care and Communication Bundle, which you will find on the IPAL-ICU resource list in the Improvement Tools section, and to think about how we can address those landmarks during the critical care course. It is really important to have the whole team attending to those same quality indicators as the days go on in the ICU to make sure that people's spiritual, emotional and communication needs are addressed.

If we use the full force of the interdisciplinary team and not get stuck on professional roles or identifications, but really look at sharing and valuing the whole process, then we are going to have the most effective support and overlap so that we do not miss any of those key clinical indicators, and that we are really providing patient-centered care the way our patients would want to be cared for. The whole team can be quite a valuable resource in all those endeavors.

Judith E. Nelson, M.D., J.D.: Those are all good points, and I think it is important for us to clarify what we mean by the key elements of palliative care in the intensive care unit. Domains for this were identified almost a decade ago by a work group supported by the Robert Wood Johnson Foundation for this purpose. After the identification of those domains, I and Kathleen and others were involved in qualitative research asking ICU patients and families to describe the kind of palliative care that they want.

There was a lot of overlap between the domains that they identified and the domains that were identified through review of the literature and professional consensus by the Robert Wood Johnson Workgroup. Patients and families stressed the importance of timely, complete and sensitive communication with their clinicians. They wanted to know about the patient's illness and about the prognosis. Another key domain described by the patients and families was to have decisions made with a focus on the patient and aligned 
with the patient's values and goals. As Kathleen brought out, there was a lot of emphasis from ICU patients and families about the dignity of the patient, the patient's comfort, the patient's personhood - meaning their uniqueness as a person and their issues of privacy - and finally, the patient's concerns that their families be cared for both in the ICU and on into the future in bereavement if that were to occur.

We describe these key components of palliative care as being, for the intensive care unit, alleviation of suffering, communication about treatments and care goals, aligning the care plan with the preferences of the patient, and helping to transition patients between different settings in a health care system where sometimes those transitions are not very smooth.

Let us now talk about the evaluation of palliative care in the ICU according to specific work processes. There have been different groups coalescing around a group of measures that might be used as a basic evaluation of palliative care, and these track the domains we have been talking about. They can be found on the IPAL-ICU website and also on a website that is maintained by the Agency for Healthcare Research and Quality, which is the National Quality Measures Clearinghouse. These measures can be looked at as ways of evaluating, tracking and, most importantly, improving palliative care in the intensive care unit. We are encouraging folks who are seeking to improve palliative care to look very specifically at their performance as they work to improve it so that they can be accountable and clear about what is successful.

I wonder if others would like to comment about what we think we could learn from efforts to improve other aspects of ICU care, some of which have been extremely successful, such as reducing central line bloodstream infections, and whether we could apply some of those lessons in the context of the ICU; or other advice on ICU quality improvement. Randy, would you like to speak to that?

J. Randall Curtis, M.D., M.P.H.: Sure. There has been some tremendous progress made, as you alluded to, in developing efforts to address quality and safety issues such as central venous catheter infections, which is a great example. One of the lessons learned is that effective quality improvement requires an interdisciplinary team effort. The team needs good interdisciplinary communication and collaboration. These teams require multiple disciplines working together as opposed to professions working in silos. Our health care system historically has been very hierarchical in its design, and one of the lessons of these quality improvement efforts is that we need to find ways to empower everybody on the team to address issues and problems that they see arising regardless of their discipline or profession.

I also think that there are some important differences between areas where we have had successes, like central venous catheter infections, and palliative care. I think that one of the reasons that the central venous catheter experience has been so successful is that a small number of clearly actionable steps were developed that clinicians could be expected to make and then held accountable for. Hand washing is the perfect example.

One of the things we have learned in palliative care is that we need to develop similar actionable steps to which we can hold clinicians accountable. As some of you know, we recently finished a randomized controlled trial of a quality improvement intervention that was not successful. One of the downfalls of the intervention was that we did not identify these clearly actionable steps that we could hold clinicians accountable for in implementing and then evaluating that intervention.

Judith E. Nelson, M.D., J.D.: Excellent points. Colleen, would you like to speak on this issue, since you have had a lot of experience in quality improvement in palliative care and you were a collaborator in the development of the Care and Communication Bundle of measures focusing on some specific work processes in ICU palliative care?

Colleen M. Mulkerin, M.S.W., LCSW: What we tried to do when we were developing those indicators was to find something that was easy to collect and not burdensome to the team, and having measures that we could define and easily agree upon was really helpful. I think ICUs that have tried to implement quality improvement measures get into a couple of different problems. One is the issue that Dr. Curtis is talking about, which is identifying actionable steps for which people can be held accountable, and trying to figure out ways to really embed these steps into the work system.

With turnover in all hospitals, especially in academic settings, you find out whether or not your systems-change effort was really durable. With a little bit of turnover, if all the folks are different, you find out if the system still stands, or was it dependent on a particular person being there to hold everybody accountable? Trying to find a system that really supports those changes so that they are truly embedded and not simply based on the personality of a particular leader is very important.

The other problem is that people see the project as a documentation project, because that is how the data are collected, instead of really thinking about the project as primarily involving clinical changes that are entailed in those core indicators. Are people really prepared clinically to conduct an effective family meeting? Do we need to do teaching around those clinical skills? What tools are we using for symptom assessment? How are we having the conversations about what people's spiritual needs are?

Sometimes our teams think we are meeting the requirements in the measures, but we find out that people do not really have the clinical skills to take each one of those indicators apart and do the teaching and the skill building that is necessary. As deceptively simple as the measures sometimes seem, it is a more complex issue to have them truly implemented.

Judith E. Nelson, M.D., J.D.: What I am hearing are some very important points that $I$ think actually can be drawn by analogy from efforts like the central line bloodstream infection initiative. There is first the point that Dr. Curtis made, which is that we simply are not going to be successful unless we are working together closely as a team. Another key lesson in my mind from the catheter-related bloodstream infection initiative is that you have to set $100 \%$ performance as your target. I think those of us who work in ICU palliative care want to be as demanding about the quality of our palliative care as we are about 
central lines. If we believe that patients' families should all have a family meeting by a specified time in the ICU, which is a very basic measure of the quality of our care, then we should not be willing to settle for less than that any more than we would settle for a significant rate of bloodstream infections. This does require team support for the target.

I think the other point that has been brought out is that we need to focus on the systems by which we provide care and not simply on telling individuals what they ought to do. One of the things we learned from the central line infection initiatives was after years of telling people to wash their hands, we realized that the only way to really make it happen was, number one, to make all the supplies readily available, and then to empower the nurses to stop procedures from being performed if the basic hygienic measures had not been followed. We can try to apply some of those lessons in the ICU, too.

As Randy Curtis has said, we need to focus very clearly on actionable steps that people can take and have them focus, as Colleen Mulkerin said, on the changes they need to make in their clinical practice to support high performance. Do others want to talk about guidance and resources for improving palliative care in the ICU, what people can look for, what is available to them, what they should target and so forth? Dr. Azoulay, could you talk a little bit about how you are approaching this in Europe?

Elie Azoulay, M.D., Ph.D.: Yes, I just wanted to say that I really share the views just expressed. There is a huge amount of literature on the attributable mortality and morbidity of bloodstream infections and catheter infections in the ICU. One thing that is convincing and effective is education based on long-term follow-up of family members, because then we know that our interventions have provided what the families needed. We reported results of family follow-up in the end-oflife conference trial in France, which we published in the New England Journal of Medicine in 2007. Now this intervention is very much used, at least in France, because people know that they can really improve their practice and meet family needs using a very simple framework.

The bundle of measures to prevent ventilator-associated pneumonia (VAP) was very efficient. Now we are trying to develop for education some bundles about how we can prepare for a family conference, so we can be sure that we are providing family members with the level of information and the amount of information they really need. We are training to provide the right amount of information for the decisionmaking process.

We are using the results observed in our studies, particularly the long-term family outcomes - for example, Mark Siegel's study of psychological morbidity of families at one year after a patient's loss, and research that Randy Curtis has published - to drive the way we are teaching.

I am sure that in teaching our nurses and our doctors, we can convince them that palliative care in the ICU is something that we can learn and improve. Using the communication skills training strategy that you have developed recently, I am sure that in the future we can really convince people that they can do better. It is important that we are using the right tools to measure symptoms, and that we are measuring something meaningful.
Judith E. Nelson, M.D., J.D.: You brought out a really important point about summarizing this growing body of evidence that demonstrates the short- and long-term impact of performance in these areas of palliative care on patients and their families and educating those who care for critically ill patients and their families about this impact as a way of emphasizing the value of improving the palliative care. I would point out that a lot of this literature (including the references that you referred to) is available in the Reference Library on the IPAL-ICU website, which is frequently updated.

The other thing mentioned was a focus on some very basic work processes, laying them out in clearly specified terms with the expectation that these will be performed, and feeding back data on performance to those of us who work in the ICU so that we can see where we are improving and where we still need to do more work.

Would people like to comment on resources that are available to support ICU palliative care improvement? Kathleen perhaps you can begin.

Kathleen Puntillo, R.N., DNSc., FAAN: Certainly. I have to think of the IPAL-ICU website that is the domain now where there are so many resources in the form of references, as you have mentioned, but also educational resources such as PowerPoint slides, pocket cards and so many other tools that are available. I cannot stress enough that this is one of the very first places that I would direct people. I know that Randy Curtis has also developed some excellent resources, and maybe he will mention some of those and how people can gain access to them.

J. Randall Curtis, M.D., M.P.H.: I agree that the IPAL-ICU website has done a nice job of collating the resources that are available. I think the kinds of resources that can be very helpful to ICUs are order sets, clinical pathways, and protocols that other institutions have developed, as well as methods to identify patients in the ICU with unmet palliative care needs. I think in terms of quality improvement tools, the IPAL-ICU website really has done a nice job of collating the available resources from many sources. We also have some of the resources we have developed on our website for the End-of-life Care Research Program, based at the University of Washington at https://depts.washington .edu/eolcare

Judith E. Nelson, M.D., J.D.: The IPAL website has a section on improvement tools where we gather good work that others have done in developing practical tools and share them openly on the web so that people do not have to reinvent wheels that have already been invented and refined by others, and we are constantly seeking new tools so that we can share them with colleagues.

The other thing that is provided on the IPAL website is an expanding series of monographs and articles by our advisory board in a section called the IPAL-ICU Portfolio, where we address some of the key issues that are faced in trying to improve palliative care in ICUs. One in particular focuses on organizing an ICU palliative care initiative. A recent one from our group relates to the surgical ICU setting. We are also going to be publishing an article in Chest about physician reimbursement for critical care services that integrate 
palliative care in the ICU, and there are others to come. So both resources and links to other resources, including Dr. Curtis's website at the University of Washington, with its research tools and other materials, are available on the website.

Dr. Azoulay, I wonder if you, or any of the others want to speak to opportunities to find resources to support improvement in ICU palliative care.

Elie Azoulay, M.D., Ph.D.: In Europe, we are also visiting the IPAL-ICU website and at this time we are also engaged in implementing information for family members on the French Society of Critical Care website. The first step would be to provide general information on the ICU, and maybe a question-answer service about the ICU for family members.

Kathleen Puntillo, R.N., DNSc., FAAN: If I might call out another one of the resources referenced on the IPAL-ICU website - it is a fairly recently published handbook by Drs. Rocker, Nelson, Azoulay and myself on end-of-life in the intensive care unit that professionals in the ICU might find helpful. It is published by Oxford University Press.

Judith E. Nelson, M.D., J.D.: Right, the Oxford Handbook of ICU Palliative Care is a nice pocket reference of which the primary editor is Graham Rocker in Canada, with contributions by many members of this panel and many other experts in the field. So the field is growing, and there are a number of readily accessible resources for people, and we hope that they will continue to be used to improve palliative care in this very important venue.

As we bring this discussion to a close, I would like to ask Dr. Siegel if he has any final comments.
Mark D. Siegel, M.D.: I think this has been a very inspiring conversation, and I think that in the background of all the work that everybody is doing here is this realization that we are changing the mindset of clinicians who practice critical care. As we put in protocols and use tools to measure our progress, it is important to remember that while all this is going on, our colleagues who do not spend all their waking moments thinking about palliative care are coming to accept us and our efforts, so that when the palliative care team comes through an ICU they are welcome, and when somebody on the team suggests the possibility that the patient has palliative care needs, there are not eyebrows raised but rather heads being nodded.

As the mindset changes, I suspect there is going to be an acceleration of change here, and I am going to predict that in the next few years that we are going to see some very dramatic improvements in the quality of palliative care being provided.

Judith E. Nelson, M.D., J.D.: Thank you. Well said. I want to thank everybody for their participation in this important discussion. We have so much expertise at this virtual table, and $I$ appreciate your taking the time from incredibly busy days to participate.

Address correspondence to: Judith E. Nelson, M.D., J.D.

Department of Medicine

Division of Pulmonary, Critical Care, and Sleep Medicine

The IPAL-ICU Project

Mount Sinai School of Medicine

One Gustave L. Levy Place

New York, NY 10029-6574

E-mail: judith.nelson@mssm.edu 